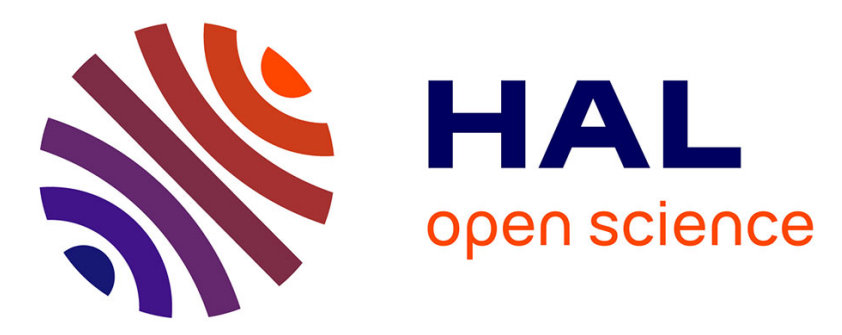

\title{
Quantification des incertitudes sur les jaugeages par exploration du champ des vitesses
}

J. Le Coz, P.M. Bechon, B. Camenen, Guillaume Dramais

\section{To cite this version:}

J. Le Coz, P.M. Bechon, B. Camenen, Guillaume Dramais. Quantification des incertitudes sur les jaugeages par exploration du champ des vitesses. La Houille Blanche - Revue internationale de l'eau, 2014, 5, pp.31-39. 10.1051/lhb/2014047 . hal-01192595

\section{HAL Id: hal-01192595 \\ https://hal.science/hal-01192595}

Submitted on 3 Sep 2015

HAL is a multi-disciplinary open access archive for the deposit and dissemination of scientific research documents, whether they are published or not. The documents may come from teaching and research institutions in France or abroad, or from public or private research centers.
L'archive ouverte pluridisciplinaire HAL, est destinée au dépôt et à la diffusion de documents scientifiques de niveau recherche, publiés ou non, émanant des établissements d'enseignement et de recherche français ou étrangers, des laboratoires publics ou privés. 


\title{
Quantification des incertitudes sur les jaugeages par exploration du champ des vitesses
}

\author{
Jérôme LE COZ11, Pierre-Marie BECHON²${ }^{2}$, Benoît CAMENEN ${ }^{1}$, Guillaume DRAMAIS ${ }^{1}$
}

\author{
1. Irstea Lyon, Unité de Recherche Hydrologie-Hydraulique, 5 rue de la Doua CS70077 69626 Villeurbanne cedex, France \\ jerome.lecoz@irstea.fr \\ 2. DREAL Rhône-Alpes, Unité Hydrométrie-Prévision des Crues, 5 Place Jules Ferry, 69006 Lyon, France \\ pierre-marie.bechon@developpement-durable.gouv.fr)
}

\begin{abstract}
RÉSUMÉ. - Evaluer la contribution des différentes sources d'erreur d'un jaugeage donné offre un outil pratique pour améliorer la stratégie de mesure. Pour répondre aux limites de la méthode proposée par la norme ISO 748, une approche généralisée est introduite pour le calcul de l'incertitude associée aux mesures de débit par exploration du champ des vitesses. Des méthodes de calcul directes sont proposées pour estimer les composantes d'incertitude liées à l'intégration verticale de la vitesse et à l'intégration transversale de la vitesse et de la profondeur. Les extrapolations de débit près des rives et dans les couches de surface et de fond sont explicitement prises en compte, de même que la répartition des verticales à travers la section. La nouvelle méthode d'analyse de l'incertitude a été appliquée à des jaugeages représentatifs de conditions de site et de protocoles contrastés. La nouvelle méthode s'avère plus polyvalente que la méthode ISO 748 et mieux adaptée à la diversité des protocoles de jaugeage. Il est possible de la mettre en œuvre dans les logiciels de dépouillement des jaugeages comme BAREME.
\end{abstract}

Mots-clés : jaugeage, incertitude, champ de vitesse, protocole.

\section{Quantification of the uncertainties related to velocity-area streamgauging data}

\begin{abstract}
Estimating the contribution of the different error sources in a given streamgauging offers a practical tool to improve the measurement strategy. To address the limitations of the method proposed by the ISO 748 standard, a generalized approach is introduced for computing the uncertainty associated with velocity-area discharge measurements. Direct computation methods are suggested for estimating the uncertainty components related to the vertical integration of velocity and to the transversal integration of velocity and depth. Discharge extrapolations to the edges and in the top/bottom layers are explicitly taken into account, as well as the distribution of the verticals throughout the cross-section. The new uncertainty analysis method was applied to streamgauging data which are representative of varied site conditions and field procedures. The new method appears to be more versatile than the ISO 748 method, and better suited to the diversity of streamgauging procedures. It is possible to implement it in discharge computation software such as BAREME.
\end{abstract}

Key-words: gauging, uncertainty, velocity area, procedure.

\section{INTRODUCTION}

Le débit des cours d'eau naturels et artificiels est un paramètre clé pour les études hydrologiques, la gestion et les décisions politiques concernant les ressources en eau, l'habitat biologique et les risques naturels. Comme tout autre type de mesure, les mesures de débit des cours d'eau sont incomplètes si l'incertitude associée, en général exprimée comme la demi-largeur de l'intervalle de confiance à $95 \%$, n'est pas fournie avec le résultat de mesure. Sans une estimation de l'incertitude de débit, l'utilisateur des données n'est pas en mesure d'évaluer les erreurs potentiellement induites dans ses conclusions, tels que l'évaluation des coûts liés aux dommages dus aux inondations, le constat d'une infraction à la réglementation sur les usages de l'eau, le dimensionnement d'un ouvrage, ou encore l'évaluation des performances d'un modèle hydrologique.

Le jaugeage par exploration du champ des vitesses [Rantz 1982 ; Herschy 1999 ; Boiten 2000] reste la technique de mesure des débits en cours d'eau la plus classique, surtout pour les petits cours d'eau. Cette méthode est régie par la norme internationale ISO 748 [ISO 2007] et sa déclinaison française NF EN ISO 748 [ISO 2009]. Outre le déploiement, sur perche ou depuis un câble, de courantomètres mécaniques (ou « moulinets »), électro-magnétiques ou acoustiques, elle couvre également les jaugeages de surface (flotteurs, radar, vidéo) et les jaugeages par ADCP fixe, verticale par verticale. Les autres techniques (ADCP mobile, dilution de traceur, volumétrie, etc.) sont fondées sur des équations de mesure différentes.

Plusieurs auteurs se sont intéressés à l'étude des sources d'erreur et à la quantification des incertitudes affectant les jaugeages par exploration du champ des vitesses, notamment [Carter et Anderson 1963 ; Fulford et Sauer 1986 ; Pelletier 1988 ; Sauer 1992 ; Fulford et Thibodeaux 1994]. Cependant, l'évaluation de l'incertitude globale d'un tel jaugeage, ainsi que du poids relatif de ses différentes sources d'erreur, reste une tâche délicate, nécessitant des hypothèses 
fortes. Les limites de la méthode proposée par la norme ISO 748 sont pointées du doigt depuis longtemps, notamment en France dans la Charte qualité de l'hydrométrie [Forray et al. 1998], qui la juge inapplicable en pratique. Il apparaît donc nécessaire de construire une méthode de calcul plus satisfaisante, qui devra être testée, confrontée aux observations et discutée en vue d'un consensus le plus large possible. Nous présentons ici les principes et l'application à des cas-tests d'une première version d'une telle approche généralisée ( méthode $\mathrm{Q}+»)$. Les résultats permettent de discuter l'adaptation de la stratégie de mesure, en termes de distribution des points et verticales de mesure, y compris la possibilité d'ajouter des verticales intercalaires sans mesure de vitesse (« méthode japonaise »).

\section{MÉTHODES DE CALCUL DES INCERTITUDES D'UN JAUGEAGE}

\section{II.1. Méthode de la norme ISO 748}

La norme [ISO 748, 2007] propose une méthode d'évaluation des incertitudes sur les jaugeages par exploration du champ des vitesses, qui a été mise en cohérence par [Herschy, 2002] avec la méthodologie et la terminologie du [GUM 2008]. Le Hydrometric Uncertainty Guidance (HUG) de 2007 [ISO/TS 25377, 2007] reprend pour l'essentiel cette méthode. Conformément au HUG, on exprimera l'incertitude finale en $\%$ du résultat $Q \pm U(Q)$ de débit au niveau de confiance $^{1}$ de $95 \%$, soit un facteur d'élargissement égal à $2: U(Q)=2 u(Q)$, où l'incertitude-type $u(Q)$ est évaluée avec l'équation suivante :

$$
\begin{aligned}
& u^{2}(Q)=\mathrm{u}_{s}^{2}+\mathrm{u}_{m}^{2}+ \\
& \frac{\sum_{\mathrm{i}=1}^{N} Q_{i}^{2}\left\{u^{2}\left(B_{i}\right)+\mathrm{u}^{2}\left(D_{i}\right)+\mathrm{u}_{p}^{2}\left(V_{i}\right)+\frac{1}{n_{i}}\left[u_{c}^{2}\left(V_{i}\right)+\mathrm{u}_{e}^{2}\left(V_{i}\right)\right]\right\}}{Q^{2}},
\end{aligned}
$$

avec dénotant partout l'incertitude-type (niveau de confiance de $68 \%$ ) exprimée en \% de la variable considérée, les débits partiels correspondant aux sous-sections ${ }^{2} \mathrm{du}$ jaugeage et dont la somme vaut $Q$, le débit total (en ajoutant les débits extrapolés aux rives). Chaque débit partiel vaut $Q_{i}=\mathrm{B}_{i} D_{i} V_{i}$, soit le produit de la largeur, de la profondeur moyenne et de la vitesse moyenne sur chaque sous-section $i$ du jaugeage. Seule la composante de vitesse normale au transect de mesure contribue au débit.

L'équation (1) fait apparaître les composantes d'incertitude suivantes :

- $u_{s}$ liée aux erreurs systématiques qui restent après le meilleur l'étalonnage des appareils de mesure de vitesse, largeur et profondeur ;

- $u_{m}$ liée aux erreurs dues au nombre limité de sous-sections (intégration transversale de la profondeur et de la vitesse) ;

- $u\left(B_{i}\right)$ et $u\left(D_{i}\right)$, liées aux erreurs aléatoires de mesure de largeur et de profondeur;

1. Un seuil de confiance de $68 \%$ fait référence à la probabilité d'être à plus ou moins un écart-type de la moyenne dans le cas d'une distribution gaussienne (et 1,96 écarts-types, arrondis à 2 pour le seuil de $95 \%$ ).

2. Dans le cas d'un calcul par la méthode de la section médiane (cas de BAREME par exemple), il y a autant de sous-sections que de verticales de mesure $(N=m)$, plus les deux sous-sections d'extrapolation aux rives. Dans le cas d'un calcul par la méthode de la section moyenne, $N=m-1$. La norme ISO 748 assimile $N$ et $m$ sans distinction.
- $u_{p}\left(V_{i}\right)$, liée aux erreurs aléatoires dues au nombre limité de mesures de vitesse sur la verticale $i$ (erreur d'intégration verticale de la vitesse);

- $u_{c}\left(V_{i}\right)$ et $u_{e}\left(V_{i}\right)$, liées aux erreurs aléatoires de mesure de la vitesse ponctuelle dues au courantomètre utilisé et à la moyenne des fluctuations turbulentes sur le temps d'exposition du courantomètre, respectivement.

Pour un nombre suffisant de verticales et donc de sous-sections de mesure $(N \geq 10)$ et un nombre constant $n$ de mesures de vitesse par verticale, si les débits partiels $Q_{i}$ sont à peu près égaux, et en supposant que les différentes composantes d'incertitude sont aussi à peu près égales sur les verticales, l'équation (1) peut être simplifiée comme suit [ISO 748, 2007] :

$$
u^{2}(Q)=\mathrm{u}_{s}^{2}+\mathrm{u}_{m}^{2}+\frac{1}{N}\left\{\begin{array}{l}
u^{2}(B)+\mathrm{u}^{2}(D)+\mathrm{u}_{p}^{2}(V) \\
+\frac{1}{n}\left[u_{c}^{2}(V)+\mathrm{u}_{e}^{2}(V)\right]
\end{array}\right\},
$$

En supposant un étalonnage correct des instruments, des valeurs typiques sont suggérées par [Herschy, 2002] et la norme [ISO 748, 2007] pour les incertitudes instrumentales : $u_{s}=1 \%, u(B)=u(D)=0,5 \%$. Il reste néanmoins souvent utile de chercher à estimer ces composantes d'incertitude à partir des résultats d'étalonnage, de vérification ou d'intercomparaison des instruments utilisés. Dans son annexe E, la norme ISO 748 fournit également des valeurs informatives pour $u_{m}$ en fonction du nombre de verticales $m$, pour $u_{p}$ en fonction de la méthode d'intégration verticale de la vitesse et du nombre de points de vitesse, pour $u_{c}$ en fonction de la vitesse mesurée et du type d'étalonnage du courantomètre (étalonnage individuel ou groupé), et pour $u_{e}$ en fonction de la vitesse mesurée, du temps d'exposition, et de la position sur la verticale. Pour en faciliter l'utilisation dans cette étude, les valeurs informatives tabulées par la norme ISO 748 ont été interpolées par des ajustements de fonctions puissance du type $u_{m}=32 \mathrm{~m}^{-0,88}$, par exemple.

Bien que le cadre d'analyse des incertitudes proposé par la norme ISO 748 soit une méthode de référence qui a le mérite d'être formalisée, son application à la diversité des mesures de débit par exploration du champ des vitesses effectuées par les services d'hydrométrie est problématique. En effet, d'un côté la méthode d'analyse d'incertitude est bien adaptée aux jaugeages "standard ", c'est-à-dire conformes à toutes les exigences de la norme ISO 748. Dans de tels cas, l'incertitude calculée se situe généralement entre $5 \%$ et $7 \%$, ce qui est l'incertitude souvent acceptée pour un jaugeage par exploration du champ des vitesses réalisé dans les bonnes conditions d'application. Cependant, pour un certain nombre de mesures " non standard ", l'incertitude des valeurs obtenues avec cette méthode apparaît irréaliste. Plus précisément, on peut identifier les inconvénients suivants de la méthode d'analyse des incertitudes de la norme ISO 748 :

1. Comme les valeurs informatives de l'annexe E pour les différentes composantes d'incertitude sont tirées d'études empiriques, elles sont spécifiques d'un type d'instrument et de certaines conditions de mesure ;

2. Les extrapolations de vitesse faites au fond, en surface et aux rives ne sont pas prises en compte dans l'analyse d'incertitude, même dans les cas où leur contribution au débit total n'est pas négligeable ;

3. Lorsque l'intégration verticale de la vitesse est effectuée point par point (méthode de distribution des vitesses), une valeur par défaut négligeable $(0,5 \%)$ est attribuée à $u_{p}$. Pour des mesures non standard (mais courantes) avec peu 
de points de vitesse et des extrapolations au fond et en surface importantes, une valeur d'incertitude-type supérieure à $0,5 \%$ devrait être considérée ;

4. La grande majorité de l'incertitude calculée provient généralement du terme $u_{m}$, qui est une fonction empirique du nombre $m$ de verticales de mesure, sans tenir compte de leur répartition spatiale par rapport à la variation transversale de la géométrie du lit et de la distribution des écoulements ;

5. L'erreur due à l'intégration temporelle dans le cas d'un débit variable au cours de la mesure n'est pas estimée, de même que les effets dus à l'écoulement transitoire (hystérésis hydraulique).

La méthode proposée a pour objectif de proposer des solutions ou des perspectives de recherche pour pallier ces différents problèmes.

\section{II.2. Incertitude liée à l'intégration verticale de la vitesse}

Trois situations se présentent en pratique : 1) application des formules algébriques à nombre de points réduit de la norme ISO 748 ; 2) méthode de distribution des vitesses (intégration en trapèzes ou graphique) ; 3) méthode par intégration (déplacement continu du courantomètre sur la verticale). La première approche n'est pas implémentée dans le logiciel BAREME, mais est utilisée par de nombreuses équipes en France (Onema, CNR, Irstea, etc.) et à l'étranger (dont USGS).

Pour ce premier cas, la norme ISO 748 fournit des valeurs d'incertitude issues d'études expérimentales pour les formules algébriques à 1,2 , et 5 points, avec $d$ la profondeur d'eau locale :

- 1 point en surface : $u_{p}=15 \%$

- 1 point (à la profondeur $0,6 d$ ) : $u_{p}=7,5 \%$

- 2 points (aux profondeurs $0,8 d$ et $0,2 d$ ) : $u_{p}=3,5 \%$

- 5 points : $u_{p}=2,5 \%$

On pourra extrapoler ces valeurs à $3 \%$ pour la méthode à 3 points (aux profondeurs $0,8 d, 0,6 d$ et $0,2 d$ ) et à $2 \%$ pour la méthode à 6 points (la norme ne propose pas de formule à 4 points). Pour la méthode du point unique en surface, il serait utile d'étudier au cas par cas l'incertitude sur le coefficient de vitesse.

Pour le deuxième cas (méthode de distribution des vitesses), au lieu de la valeur forfaitaire de la norme ISO $748(0,5 \%)$, souvent sous-estimée en pratique, nous proposons une méthode de calcul direct du terme $u_{p}$ qui considère explicitement les erreurs d'interpolation (sur la zone explorée) et d'extrapolation (sur les zones aveugles de fond et en surface) des vitesses. A partir de l'hypothèse d'une distribution de probabilité rectangulaire [GUM, 2008] pour les erreurs d'interpolation et d'extrapolation (voir le détail des calculs dans [Le Coz et al., 2012a] et l'erratum sous presse), on aboutit à l'équation suivante :

$$
u_{p}^{2}\left(V_{i}\right)=\frac{1}{12 q_{i}^{2}}\left\{\begin{array}{l}
\sum_{\mathrm{k}=1}^{n_{i}-1}\left[\left(v_{\mathrm{i}, \mathrm{k}}-v_{\mathrm{i}, \mathrm{k}+1}\right)\left(d_{\mathrm{i}, \mathrm{k}}-d_{\mathrm{i}, \mathrm{k}+1}\right)\right]^{2} \\
+\mathrm{E}^{2}\left(q_{\mathrm{i}, \text { haut }}\right)+\mathrm{E}^{2}\left(q_{\mathrm{i}, \text { bas }}\right)
\end{array}\right\},
$$

où $q_{i}$ désigne le débit par unité de largeur (ou P.U.) de la sous-section $i, v_{\mathrm{i}, \mathrm{k}}$ est la vitesse ponctuelle mesurée à la cote $d_{\mathrm{i}, \mathrm{k}}$ au-dessus du fond, le premier terme sous l'accolade est l'erreur maximale estimée sur le P.U. partiel de la zone explorée, et $E\left(q_{\mathrm{i}, \text { haut }}\right)$ et $E\left(q_{\mathrm{i}, \text { bas }}\right)$ sont les erreurs maximales considérées sur les P.U. partiels des couches extrapolées en haut et en bas.
Ces deux erreurs maximales peuvent être estimées à partir des options extrêmes d'extrapolation du profil de vitesse au fond et en surface (cf. Figure 1). Ainsi, l'erreur maximale de débit de fond est simplement calculée comme suit, puisque les pires des cas sont supposés être un profil de vitesse linéaire à zéro au niveau du lit, et un profil de vitesse constante :

$$
E\left(q_{\mathrm{i}, \mathrm{bas}}\right)=\frac{1}{2} v_{i, 1} d_{i, 1}
$$

avec $v_{\mathrm{i}, 1}$ la vitesse mesurée la plus basse et $d_{\mathrm{i}, 1}$ la hauteur de la couche de fond extrapolée.

De même, l'erreur maximale de débit de surface peut être estimée, soit à partir de différentes options d'extrapolation en surface (cf. Figure 1), soit plus simplement en supposant que dans le pire des cas, la vitesse peut varier linéairement de plus ou moins $20 \%$ entre le plus haut point de mesure et la surface :

$$
E\left(q_{\mathrm{i}, \text { haut }}\right)=0,20 v_{i, n}\left(h-d_{i, n}\right)
$$

avec $v_{\mathrm{i}, \mathrm{n}}$ la vitesse mesurée la plus haute et $h-d_{\mathrm{i}, \mathrm{n}}$ la hauteur de la couche de surface extrapolée. C'est cette dernière équation qui a été retenue pour l'implémentation dans le logiciel BAREME.

Le cas de la méthode par intégration est traité de la même façon que la méthode de distribution des vitesses avec les modifications suivantes :

- le nombre $n_{i}$ de mesures de vitesse indépendantes sur la verticale $i$ est égal au nombre de cycles complets (descente/

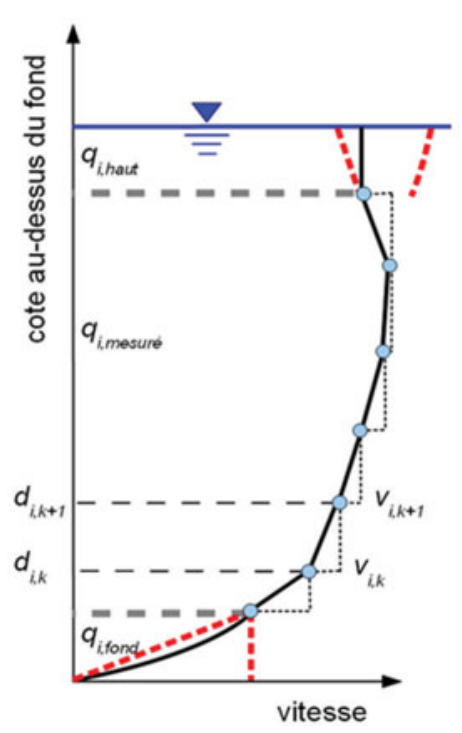

Figure 1 : Principe du calcul de l'erreur maximale sur la vitesse moyennée sur la verticale, erreur due à l'échantillonnage des vitesses dans la couche mesurée et aux choix d'extrapolation dans les couches de fond et de surface. Dans la couche supérieure, les options illustrées sont l'extrapolation constante (ligne noire pleine), l'ajustement en loi puissance et l'ajustement linéaire sur les deux points les plus hauts (lignes pointillées rouges). Dans la couche inférieure, les options illustrées sont l'ajustement en loi puissance (ligne noire pleine), et les extrapolations constante et linéaire (lignes pointillées rouges). 
remontée) du courantométre (en général 1, et 2 selon préconisations ISO 748);

- l'erreur due à l'intégration verticale dans la zone explorée est supposée négligeable en raison de l'intégration physique effectuée continuement ;

- $E\left(q_{\mathrm{i}, \text { haut }}\right)$ est calculé avec la vitesse intégrée plutôt que la vitesse $v_{\mathrm{inn}}$ puisque l'extrapolation constante est généralement basée sur cette vitesse moyenne ;

- pour calculer $E\left(q_{\mathrm{i}, \text { bas }}\right)$, la vitesse de point de fond $v_{\mathrm{i}, 1}$ est soit mesurée, soit déduite de la vitesse intégrée par un coefficient supplémentaire, conformément au mode de calcul du débit choisi.

\section{II.3. Incertitude liée à l'intégration transversale de la profondeur et de la vitesse}

Une nouvelle méthode est également proposée pour estimer l'incertitude due aux erreurs d'intégration transversale des profondeurs et des vitesses moyennes sur la profondeur. $\mathrm{Au}$ lieu du terme unique $u_{m}$ de la norme ISO 748, les incertitudes d'intégration transversale sont calculées séparément sur la profondeur moyenne (composante $u_{m}\left(D_{i}\right)$ ), et sur la vitesse moyenne (composante $u_{m}\left(V_{i}\right)$ ) sur chaque sous-section $i$ du jaugeage. Cela conduit à l'Equation suivante ${ }^{3}$, modifiée à partir de l'Equation (1) :

$$
u^{2}(Q)=\mathrm{u}_{s}^{2}+\frac{\sum_{\mathrm{i}=0}^{\mathrm{N}+1} Q_{i}^{2}\left\{\begin{array}{l}
u^{2}\left(B_{i}\right)+\mathrm{u}^{2}\left(D_{i}\right)+\mathrm{u}_{p}^{2}\left(V_{i}\right)+\mathrm{u}_{m}^{2}\left(D_{i}\right) \\
+\mathrm{u}_{m}^{2}\left(V_{i}\right)+\frac{1}{n_{i}}\left[u_{c}^{2}\left(V_{i}\right)+\mathrm{u}_{e}^{2}\left(V_{i}\right)\right]
\end{array}\right\}}{Q^{2}},(
$$

Pour évaluer, l'incertitude sur la profondeur moyenne $D_{i}$, on estime l'erreur maximale $E\left(D_{i}\right)$ due à l'intégration transversale, en supposant une distribution uniforme rectangulaire [GUM, 2008]. Ainsi :

$$
u_{m}\left(D_{i}\right)=\mathrm{E}\left(D_{i}\right) /\left(2 \sqrt{3} D_{i}\right)
$$

3. Noter en outre que les sous-sections de rives sont incluses dans l'Equation (6), contrairement à l'Equation (1), avec les indices $i=0$ et $i=N+1$.
Des approches similaires ont été récemment étudiées par d'autres auteurs, comme [Olivier et al. 2009] et [Kiang et al. 2009], la différence fondamentale se situant dans l'estimation de $E\left(D_{i}\right)$. Afin d'éviter un certain nombre d'effets indésirables (voir [Le Coz et al. 2012a]), nous proposons de fonder cette estimation sur l'amplitude maximale de variation de la section mouillée, calculée à partir de scénarios extrêmes sur la variation transversale du fond (cf. Figure 2). Ces scénarios peuvent être évalués à l'aide d'une pente transversale du fond maximale définie par l'utilisateur (angle $\alpha$ ). Cette pente doit inclure l'effet des irrégularités du fond comme des mouilles ou des blocs par exemple, et peut être fixée par défaut à la pente maximale vue dans la bathymétrie du jaugeage. Son estimation peut aussi prendre en compte des propriétés physiques comme l'angle de stabilité du matériau granulaire constituant le fond (on considère souvent $40^{\circ}$ pour du sable immergé). Les sous-sections de rive sont traitées de la même façon que les autres sous-sections.

On suit une approche similaire pour évaluer $u_{m}\left(V_{i}\right)$, l'incertitude sur la vitesse moyenne $V_{i}$ sur la sous-section $i$ :

$$
u_{m}\left(V_{i}\right)=\mathrm{E}\left(V_{i}\right) /\left(2 \sqrt{3} V_{i}\right)
$$

Pour établir les pires scénarios de distribution transversale des vitesses, le profil de vitesse est interpolé sur la base des profils de profondeur extrêmes calculés précédemment. Nous utilisons une interpolation en $\sqrt{d}$ pour calculer les variations des vitesses, ce qui peut être justifié par l'hypothèse d'un nombre de Froude [Fulford et Sauer, 1986] ou d'un coefficient de Chézy [Boiten, 2000] [ISO/ TR 9823, 1990] constant ou variant linéairement, le rayon hydraulique étant approché par la profondeur locale $d$. Les profils transversaux de vitesse $\mathrm{min} / \mathrm{max}$ ainsi obtenus peuvent être intégrés linéairement sur la sous-section pour calculer l'écart maximal de vitesse moyenne $E\left(V_{i}\right)$. La discontinuité des profils de vitesse aux bords des soussections n'est pas un problème puisque le but n'est pas de simuler des distributions de vitesse réalistes, mais d'estimer les variations maximales possibles de la vitesse moyenne sur la sous-section de jaugeage considérée. a

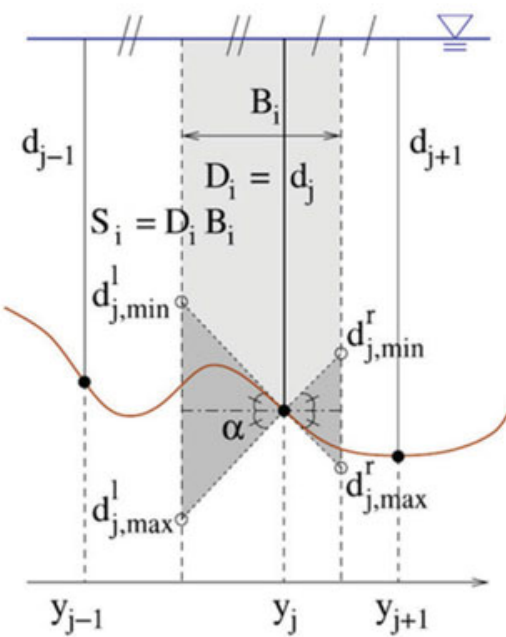

b

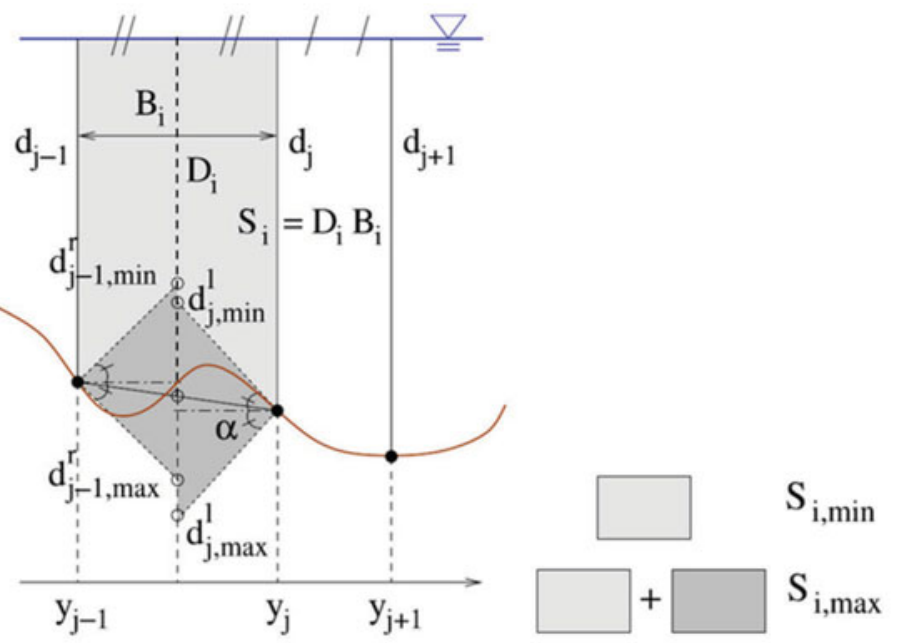

Figure 2 : Principes du calcul de la composante d'incertitude liée à l'intégration transversale de la profondeur, à partir d'un angle $\alpha$ de variation maximale de la pente du fond. Evaluation des sections mouillées minimale et maximale pour la méthode de la section médiane ( $a$, rectangles) et la méthode de la section moyenne (b, trapèzes). 
Les sous-sections aux rives doivent cette fois-ci être traitées différemment des autres puisque la vitesse moyenne y est extrapolée à l'aide d'un coefficient de rive $k_{r}: V_{\text {rive }}=\mathrm{k}_{r} \bar{v}_{r}$. En considérant les valeurs extrêmes 0,67 et 0,91 pour $k_{r}$ en s'inspirant de la norme ISO 748, il vient simplement : $E\left(V_{\text {rive }}\right) \approx 0,24 \bar{v}_{r}$.

On pourra retenir les avantages suivants de cette nouvelle méthode d'estimation des composantes d'incertitude liées à l'intégration transversale des profondeurs et vitesses :

- la méthode de calcul est symétrique à la fois pour la méthode de la section médiane (Fig.2a, méthode des rectangles) et pour la méthode de la section moyenne (Fig.2b, méthode des trapèzes) ;

- elle permet de séparer les composantes d'incertitude sur la profondeur (ou la section mouillée) et sur la vitesse, contrairement aux travaux de [Olivier et al. 2009] et [Kiang et al. 2009] ;

- elle traite explicitement le cas des sous-sections de rives, ce qui permet d'évaluer leur contribution à l'incertitude finale ;

- les hypothèses faites sur les variations transversales de profondeur et de vitesse sont interprétables d'un point de vue physique, et donc vérifiables et améliorables notamment en ajoutant de la connaissance sur le site de mesure.

\section{EVALUATION, EXPLOITATION ET DÉVELOPPEMENT DE LA MÉTHODE}

\section{III.1. Application à des cas-tests variés}

La nouvelle méthode a été appliquée à un ensemble de jaugeages variés en termes de conditions de mesure et de protocole de déploiement. Nous présentons ici deux cas emblématiques d'une section naturelle et d'un canal maçonné à section rectangulaire (Figure 3). D'autres cas sont étudiés dans [Le Coz et al., 2012a] : jaugeage de crue à 6 points sur l'Ardèche à Sauze, jaugeage par intégration sur la Doller à Burnhaupt, jaugeage au micro-moulinet dans un canal de laboratoire à lit composé. Bien entendu, la méthode nécessite encore des confrontations à de nombreux autres jaugeages pour que sa validité fasse l'objet d'un consensus large.

Le premier exemple, l'Arc-en-Maurienne à Saint-Avre (Figure 3, haut), est typique des conditions de faible débit dans une rivière de montagne avec un grand rapport d'aspect, ou ratio largeur au miroir sur profondeur moyenne, qui est pour ce jaugeage proche de 100. Plus généralement, de nombreuses sections de jaugeage en cours d'eau naturel présentent aussi de grands rapports d'aspect. Les mesures ont été effectuées à l'aide d'un courantomètre électromagnétique (Ott Nautilus) monté sur une perche, également utilisée pour lire les profondeurs. La procédure ISO 748 à 3 points de vitesse a été appliquée sur les 20 verticales, sauf sur la dernière où la procédure à 1 point a été préférée en raison du manque de profondeur. Les débits extrapolés aux rives sont négligeables par rapport au débit total, comme souvent sur les sections naturelles. Comme on peut le voir sur l'histogramme de la Fig. 3 (haut), cette mesure répond aux exigences de la norme ISO 748 puisque tous les sous-débits sont inférieurs à $10 \%$ du débit total.

Pour ce jaugeage standard de l'Arc, sur section naturelle et respectant les préconisations de la norme ISO 748, la nouvelle méthode fournit une incertitude globale $(U(Q)=7,0 \%)$ qui est en pratique proche de celle fournie par la méthode de la norme ISO $748(U(Q)=5,2 \%)$. La distribution des variances (voir le camembert en Figure 3, haut) est intéressante car elle présente le poids respectif des différentes sources d'incertitude. Selon la nouvelle méthode, les contributions des termes d'intégration transversale $u_{m}(D)$ et $u_{m}(V)$ sont équivalentes entre elles, et représentent à elles deux $95 \%$ de l'incertitude totale. Selon la méthode ISO 748, le terme $u_{m}$ pèse pour $77 \%$ (en variance) de l'incertitude totale. La nouvelle méthode se comporte ainsi de façon similaire à celle de la norme ISO 748, pour un cas de jaugeage classique sur section naturelle large. Les résultats confirment qu'ici, c'est l'échantillonnage transversal, lié au nombre et à la position des verticales de mesure, qui est le facteur limitant en termes d'incertitude sur le débit jaugé.

Le deuxième cas d'application, un petit canal d'irrigation à Gignac (Figure 3, bas), fournit l'exemple typique d'un échantillonnage de vitesse dense sur un nombre limité de verticales à travers une étroite section rectangulaire, qui présente un rapport d'aspect bien plus faible, proche de 1 (1,34 $\mathrm{m}$ de largeur pour $1 \mathrm{~m}$ de profondeur). La méthode de distribution des vitesses a été suivie avec 10 positions tous les $10 \mathrm{~cm}$ en plus d'une position aussi proche du fond que possible $(1 \mathrm{~cm})$ en utilisant un micro-moulinet. En raison de la faible largeur de section et de la durée de mesure des vitesses, 6 verticales seulement ont été disposées uniformément, en s'approchant au plus près possible des bords. En conséquence, la plupart des sous-débits sont supérieurs à $15 \%$ du débit total, et les débits extrapolés aux bords représentent des contributions importantes (13\% et $8 \%$ ) au débit total. Près de chaque rive, la variation de vitesse est bien reproduite avec un coefficient de rive $k_{r}=0,86$, ce qui est cohérent avec des parois assez lisses. Cette mesure est ainsi très loin d'être standard, au sens de la norme ISO 748.

Cette fois-ci, les deux méthodes fournissent des valeurs d'incertitude très différentes : $U(Q)=13,4 \%$ pour la méthode ISO 748, contre $U(Q)=3,4 \%$ seulement pour la nouvelle méthode. La première valeur apparaît disproportionnée par rapport aux comparaisons qui ont été effectuées avec des mesures de débit sur déversoir étalonné, avec des écarts constatés de 1'ordre de $3 \%$. Elle provient directement du nombre limité (6) de verticales, avec un poids écrasant du terme $u_{m}$ dans l'incertitude totale (98\%). La nouvelle méthode fournit donc une valeur d'incertitude apparemment faible, mais bien plus réaliste. La distribution des sources d'erreur est également bien plus crédible : l'incertitude d'intégration transversale est jugée négligeable comme attendu pour une section très régulière de géométrie connue, tandis que l'incertitude dominante provient des extrapolations de débit aux rives, suivie de loin par l'incertitude d'intégration verticale des vitesses.

Le seul paramètre supplémentaire dont la valeur doit être estimée par l'utilisateur pour le calcul d'incertitude est l'angle maximal de la pente transversale du fond, noté $\alpha$. En considérant la nature du fond (graviers pour l'Arc, béton pour le canal de Gignac) ainsi que notre connaissance de la géométrie (reconnaissance des irrégularités en cours de jaugeage à pied et variations visibles dans la bathymétrie de l'Arc, profil calibré du canal de Gignac), une valeur d'angle $\alpha$ de $15^{\circ}$ pour l'Arc et de $10^{\circ}$ pour Gignac a été retenue. La sensibilité du résultat d'incertitude à la valeur de $\alpha$ est d'autant plus forte que le rapport d'aspect de la section est grand. Par exemple, en considérant un angle $\alpha$ de $40^{\circ}$, valeur disproportionnée pour les deux cas, l'incertitude finale pour Gignac ne varie quasiment pas $(3,6 \%$ au lieu de 3,4\%) tandis qu'elle atteint la valeur peu réaliste de $18,9 \%$ pour l'Arc, au lieu de 7,0\%. Une analyse de sensibilité plus complète est présentée dans [Le Coz et al., 2012a]. 

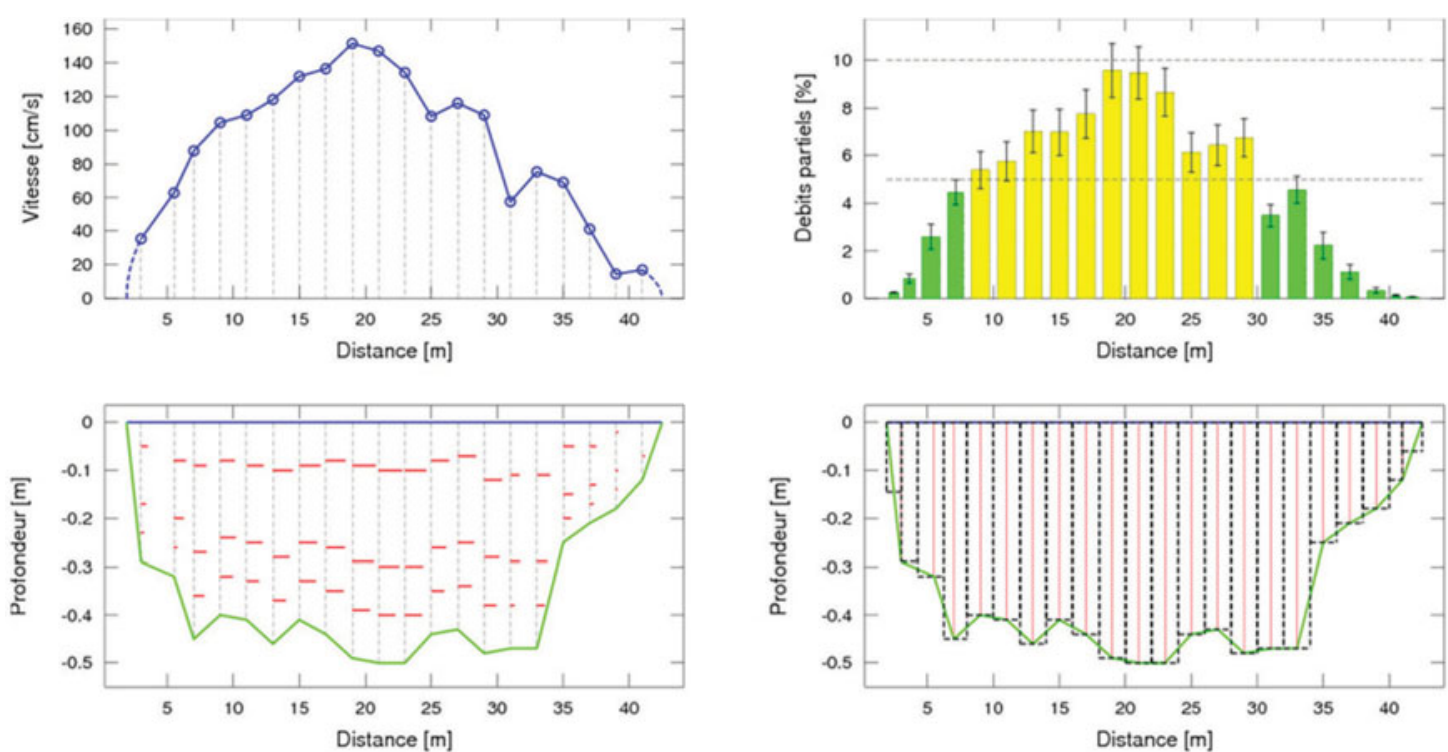

Nom de fichier : Arc060404.da Courantometre : Nautilus

Integration verticale : algebrique (1-2-3 pts) + point par poin

Integration laterale : section mediane (rectangles)
Coefficients de rugosite: $m_{1}=6, m_{g}=2, m_{d}=2$

Debit total $=15.503 \mathrm{~m} 3 / \mathrm{s}$

Surface mouillee $=15.24 \mathrm{~m}$

nne $=1.02 \mathrm{~m} / \mathrm{s}$

Incertitude $Q_{+}=7.0 \%$ (angle fond $=15$ deg.)

Incertitude ISO748 $=5.2 \%\left(20\right.$ verticales, poids $\left.u_{m}: 77.4 \%\right)$
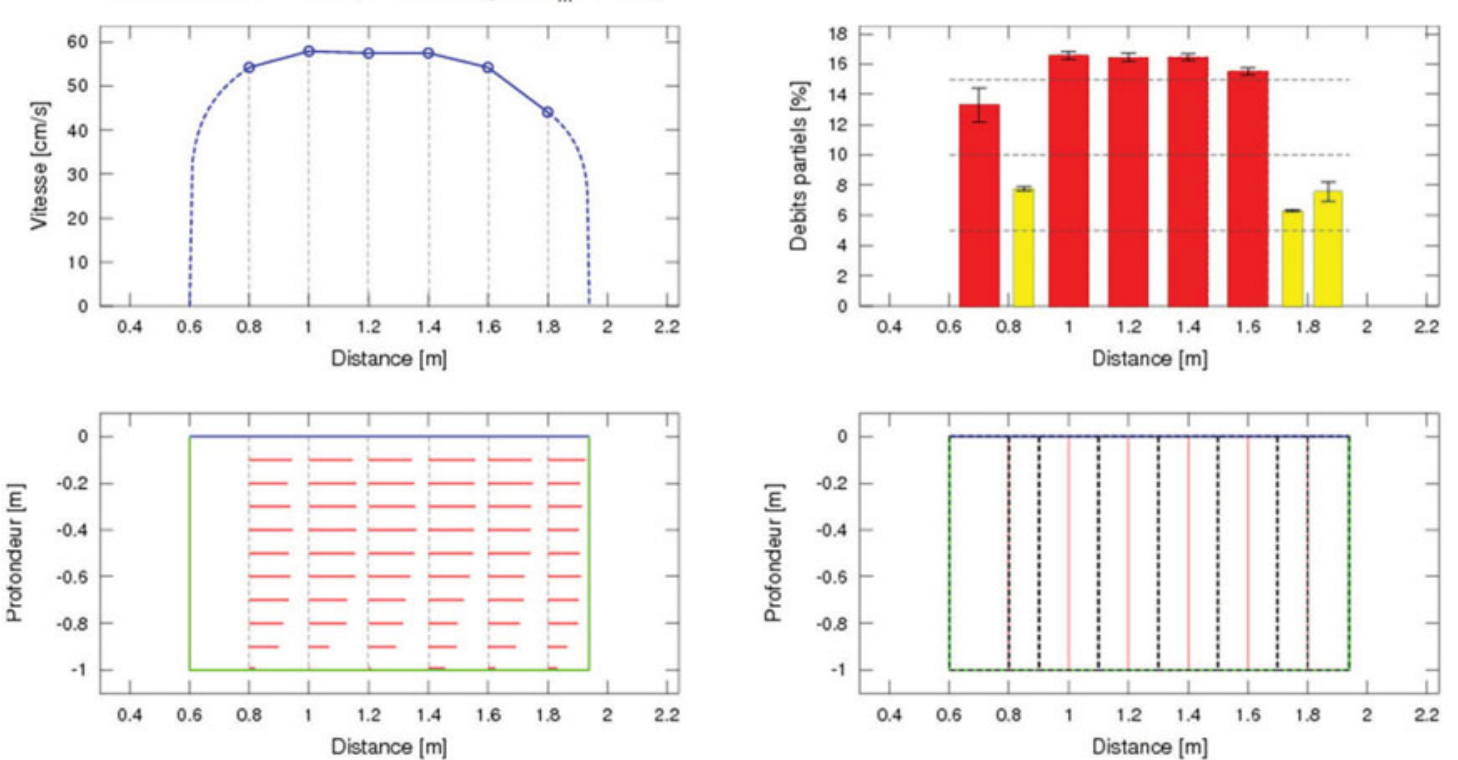

Nom de fichier : gignac2.dat

Courantometre : Flowtracke

Integration verticale : algebrique (1-2-3 pts) + point par point

Integration laterale : section mediane (rectangles)

Coefficients de rugosite: $m_{1}=6, m_{g}=6, m_{d}=6$

Debit total $=0.699 \mathrm{~m} 3 / \mathrm{s}$

Vitesse moyenne $=0.52 \mathrm{~m} / \mathrm{s}$

Incertitude $\mathrm{Q}_{+}=3.4 \%$ (angle fond $=10$ deg.)

Incertitude ISO748 $=13.4 \%$ ( 6 verticales, poids $u_{m}: 97.5 \%$ )
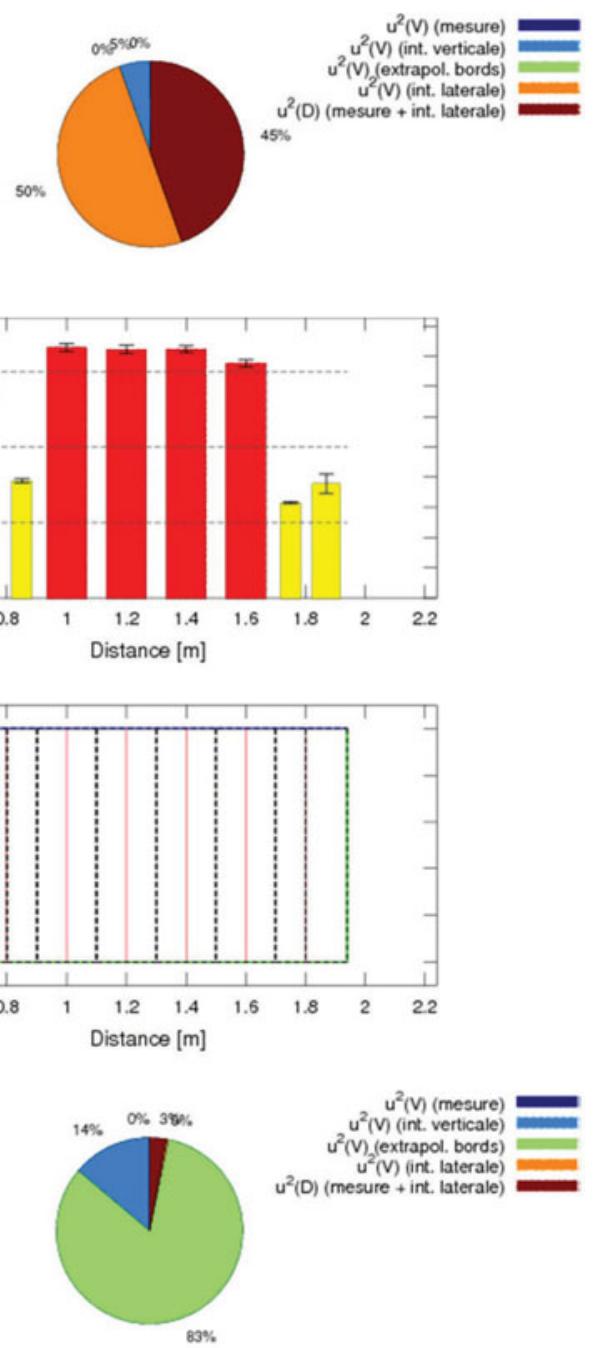

$u^{2} u^{2}(V)$ (mesure)

$u^{2}(V)$ (extrapol. bords)

$u^{2}(V)$ (int. laterale) $u^{2}$ (D) (mesure + int. laterale)

Figure 3 : Exemples d'application de la méthode à deux jaugeages très différents : jaugeage en section naturelle très plate (haut, Arc-en-Maurienne à St-Avre) avec des incertitudes d'intégration transversale dominantes (en orange-marron dans le camembert) ; jaugeage en canal étroit (bas, canal de Gignac) avec des incertitudes liées aux extrapolation de bords (en vert dans le camembert) dominantes. Le poids des erreurs d'intégration verticale de la vitesse est indiqué en bleu. 


\section{III.2. Amélioration du processus de mesure}

Par rapport à la méthode de départ de la norme ISO 748, la nouvelle méthode permet donc une analyse plus fine et plus fiable des incertitudes dues à l'échantillonnage spatial des vitesses et des profondeurs, verticalement et transversalement. Il devient alors possible d'évaluer et d'améliorer le processus de mesure, en termes de nombre et de disposition des verticales et des points de vitesse à travers la section de jaugeage. A ce titre, le rapport d'aspect de la section (ratio largeur sur profondeur moyenne) apparaît comme un critère prépondérant pour ajuster la stratégie d'échantillonnage spatial [Le Coz et al., 2011]. On peut ainsi distinguer deux configurations de site très distinctes mais recouvrant la plupart des situations de jaugeage rencontrées en pratique. Les recommandations indiquées ci-après peuvent être évaluées au cas par cas avec la nouvelle méthode d'analyse des incertitudes.

Dans le cas d'une section naturelle présentant un rapport d'aspect élevé ( $>10$ typiquement), il est recommandé d'utiliser au moins 11 à 15 verticales afin de limiter le nombre de sous-sections représentant plus de $10 \%$ du débit total, et faire en sorte que le débit partiel ne dépasse $15 \%$ sur aucune sous-section; il est toutefois acceptable de retirer des verticales sur des portions de la section où la profondeur et la vitesse sont constantes. La méthode algébrique à trois points sur la verticale apparaît, si l'écoulement est uniforme, comme un optimum pour réduire l'incertitude de mesure tout en limitant la durée du déploiement.

Pour une section étroite et profonde (type canal de dérivation), présentant donc un faible rapport d'aspect $(<10$ typiquement), il est souvent nécessaire de réduire le nombre de verticales à cause de l'encombrement du matériel et de la distance minimale du courantomètre aux parois. On essaiera de maintenir un nombre minimal de six verticales, tout en décrivant au mieux la variation de vitesse près des bords. La géométrie d'un canal étant généralement très régulière, on disposera les verticales en fonction des variations de vitesse sur la largeur. En revanche, il est utile d'augmenter le nombre de points sur chaque verticale pour réduire les erreurs liées à des profils verticaux de vitesse plus irréguliers qu'en section large (influence des parois). En recommandant pour cette configuration un minimum de 5 points sur chaque verticale, on aboutit à un minimum de 30 points de vitesse sur la section, comme pour la configuration en section naturelle.

Une amélioration intéressante du processus de mesure consiste à ajouter des verticales intercalaires où seule la profondeur est mesurée tandis que la vitesse moyenne est interpolée à partir des mesures de vitesse adjacentes (cf. Fig. 4). Cette méthode est inspirée de la "méthode japonaise » décrite dans la notice du courantomètre acoustique FlowTracker (SonTek), en ajoutant une interpolation des vitesses en $\sqrt{d}$ avec $d$ la profondeur locale (voir la justification en Section II.3). L'avantage découle des idées suivantes : le profil bathymétrique étant généralement moins lisse et plus variable que le profil transversal de vitesse moyenne, il gagne à être échantillonné plus finement ; le temps nécessaire pour mesurer la profondeur de l'écoulement est négligeable par rapport au temps nécessaire pour mesurer la vitesse, en raison du nombre de points et du temps d'exposition requis. L'idée est donc de mieux décrire la géométrie sans perdre trop de temps.
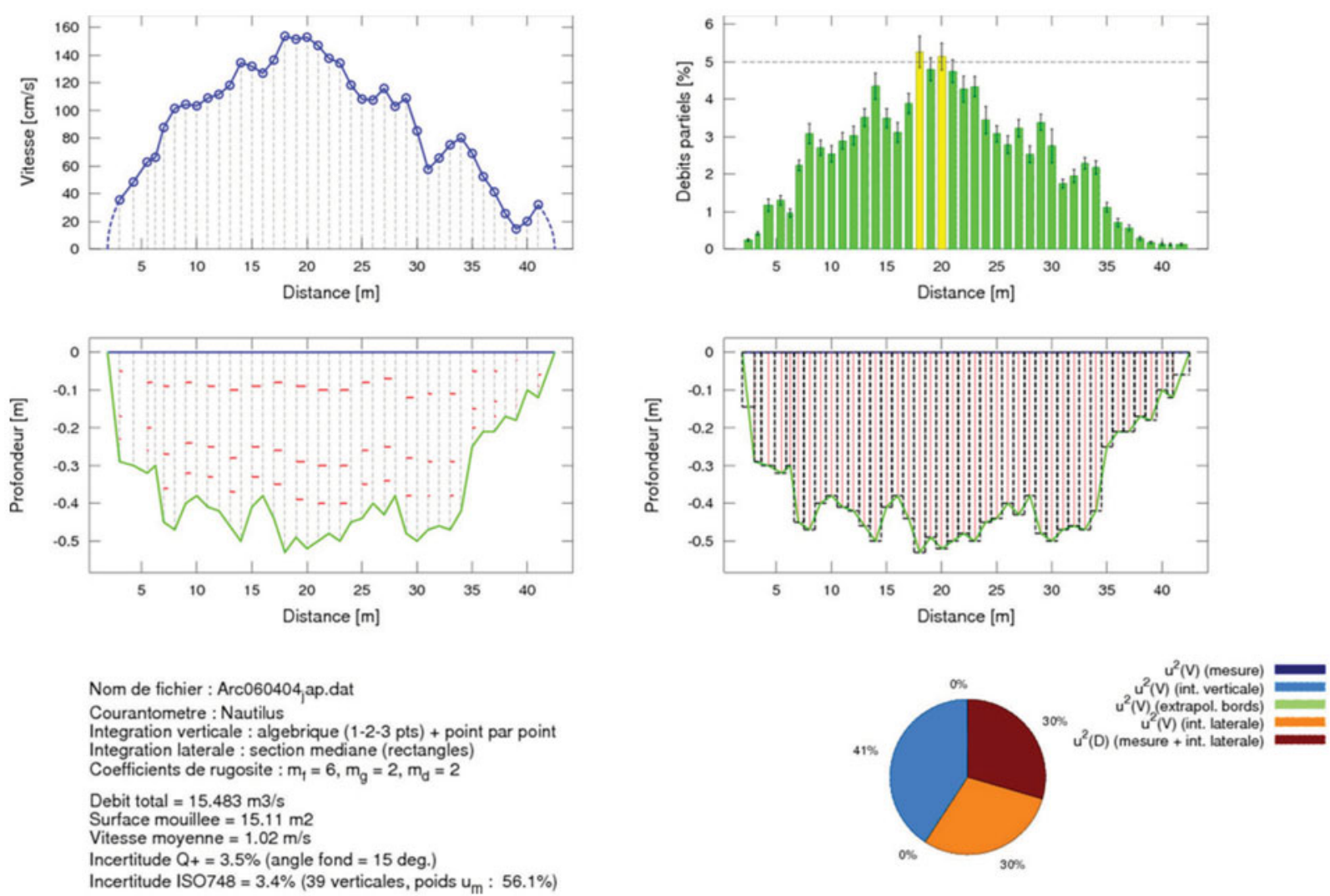

Figure 4 : Application de la méthode des verticales intercalaires au jaugeage de l'Arc-en-Maurienne à St-Avre. L'incertitude globale évaluée par la nouvelle méthode est réduite à 3,5\% au lieu de 7,0\% sans les verticales intercalaires. Les codes couleur du camembert sont les mêmes que pour la Figure 3. 
Sur une verticale intercalaire $i$, l'incertitude $u_{p}\left(V_{i}\right)$ sur la vitesse moyenne interpolée provient des erreurs du modèle d'interpolation et de l'incertitude des vitesses mesurées sur les verticales adjacentes utilisées dans l'interpolation. En supposant que ces sources d'erreur sont indépendantes, les variances correspondantes peuvent être ajoutées. Comme le profil transversal de vitesse est généralement convexe, l'erreur d'interpolation maximale peut être estimée égale à $\left(V_{i+1}-V_{i-1}\right) / 2$. Par conséquent, en supposant une densité de probabilité rectangulaire [GUM, 2008] pour l'erreur d'interpolation des vitesses, on obtient :

$$
u_{p}^{2}\left(V_{i}\right)=\frac{\left(V_{i+1}-V_{i-1}\right)^{2}}{12}+u_{p}^{2}\left(V_{i+1}\right)+u_{p}^{2}\left(V_{i-1}\right),
$$

Cette méthode de mesure a été testée sur le jaugeage de l'Arc présenté précédemment, en ajoutant une verticale intercalaire entre deux verticales de mesure des vitesses (voir Fig. 4). L'incertitude globale évaluée par la nouvelle méthode est significativement réduite, à 3,5\% au lieu de $7,0 \%$ sans les verticales intercalaires. Cette valeur, proche de ce que la norme ISO 748 indique pour 39 verticales $(3,4 \%)$, peut sembler un peu sous-estimée, du fait de la non-prise en compte de certaines erreurs instrumentales et environnementales (discutées plus bas). Néanmoins, ce résultat montre l'importante réduction des incertitudes liées à l'échantillonnage spatial : la contribution des termes d'intégration transversale $u_{m}(D)$ et $u_{m}(V)$ est passée de $95 \%$ à $60 \%$ de l'incertitude totale. Il deviendrait alors intéressant d'augmenter le nombre de points sur la verticale, puisque le terme $u_{p}(V)$ n'est plus négligeable et pèse pour $40 \%$ de la variance totale. A noter que même pour $\alpha=40^{\circ}$, l'incertitude calculée resterait limitée à $8,2 \%$.

\section{III.3. Perspectives de développement de la méthode}

Comme déjà indiqué, certaines erreurs instrumentales et environnementales sont sans doute imparfaitement prises en compte dans la nouvelle équation des incertitudes (Equation 6), ou dans les valeurs attribuées aux termes correspondants. Les effets de site et d'opérateur, souvent importants, sont très difficiles à mettre en équation, d'où l'intérêt de les évaluer par des comparaisons interlaboratoires [Dramais et al., 2013]. Du côté des erreurs instrumentales, le terme $u_{s}$ représentant l'incertitude liée aux effets systématiques imparfaitement corrigés par les étalonnages, mais aussi à ceux liés à la méthode de jaugeage, agit comme une valeur plancher de l'incertitude finale : en prenant par défaut $u_{s}=1 \%$, l'incertitude finale $U(Q)$ ne peut pas être calculée inférieure à $2 \%$. Il est clair que dans de nombreux cas, cette valeur sera sous-estimée, d'où l'intérêt de la ré-évaluer à partir d'étalonnages, de vérifications et de confrontation à des mesures de référence d'incertitudes maîtrisées et suffisamment réduites, ce qui reste souvent problématique. Les valeurs informatives indiquées par la norme ISO 748 pour les termes $u_{c}$ et $u_{e}$ liés à la mesure ponctuelle de la vitesse seraient aussi à adapter en fonction des performances du modèle de courantomètre utilisé $\left(u_{c}\right)$ d'une part, et de sa cadence d'échantillonnage temporel des vitesses, de la durée d'exposition et de l'intensité des fluctuations turbulentes de l'écoulement $\left(u_{e}\right)$ d'autre part. De plus, les erreurs d'angle liées au déploiement de la perche, du câble et du courantomètre mériteraient une évaluation plus poussée car elles peuvent entraîner des incertitudes importantes sur les profondeurs, les positions des vitesses et leur projection perpendiculairement à la section de jaugeage.
Typiquement, une erreur importante sur la mesure de vitesse souvent ignorée provient du déploiement du courantomètre dans trop peu d'eau ou trop près d'obstacles. Dans l'exemple du canal de Gignac, les points de vitesse réalisés à $1 \mathrm{~cm} \mathrm{du}$ fond pour minimiser le débit extrapolé au fond présentent des valeurs douteuses par rapport au reste du profil hydraulique. Il est alors probable que l'incertitude instrumentale engendrée, et non prise en compte dans la méthode de calcul, soit supérieure au gain réalisé sur l'extrapolation du débit de fond. La nouvelle méthode ne permet pour l'instant pas d'évaluer ce type d'effets. Rappelons cependant que la norme ISO 748 préconise qu'un courantomètre mécanique (moulinet) ne doit pas être utilisé lorsque le tirant d'eau est inférieur à quatre fois le diamètre de l'hélice ou du corps du moulinet. De même, les courantomètres électromagnétiques ou acoustiques ne doivent pas être utilisés lorsque le tirant d'eau est inférieur à trois fois la dimension verticale du capteur. Dans tous les cas, aucune partie du courantomètre ne doit dépasser la surface de l'eau. Ces règles sont plus souvent violées qu'on ne pourrait le croire.

Jauger par exploration du champ des vitesses dans une section trop peu profonde peut ainsi entraîner de grandes erreurs, surtout quand en plus le fond est meuble (sable) et se déforme sous l'effet du sillage de la perche et de l'opérateur. En outre, quand la méthode de distribution des vitesses est utilisée, avec un petit nombre de points de vitesse sur la verticale, le poids des incertitudes dues aux extrapolations de débit au fond et en surface devient souvent très important. Dans de tels cas, il est donc recommandé de recourir à une technique de jaugeage plus adaptée (dilution, volumétrie, ouvrages hydrauliques...).

Enfin, dans le cas d'un débit qui varie sensiblement pendant la durée du jaugeage, deux erreurs distinctes d'ordre environnemental peuvent se manifester : l'erreur d'intégration temporelle de vitesses et profondeurs non-stationnaires, et l'hystérésis hydraulique qui entraîne un écart de la relation hauteur-débit réelle au moment du jaugeage, par rapport aux conditions de régime permanent pour laquelle est établie la courbe de tarage que l'on cherche à documenter avec le jaugeage [Le Coz et al., 2012b]. Il est possible d'évaluer simplement l'incertitude additionnelle engendrée par ces deux effets, en considérant la variation maximale possible de débit sur la durée du jaugeage selon la courbe de tarage, et en utilisant la formule de [Jones, 1916] pour corriger le biais entraîné par l'hystérésis hydraulique et évaluer l'incertitude de la correction [Le Coz et al., 2012a]. Implémenter ces deux composantes d'incertitude dans le calcul serait intéressant pour quantifier ce qu'on gagne à jauger plus rapidement les écoulements transitoires, en cas de crue rapide notamment. Il est probable que parfois, ajouter des verticales, des points de vitesse et du temps d'exposition produit un gain d'incertitude qui est annihilé voire dépassé par les deux erreurs d'intégration temporelle et d'hystérésis. De plus, pour les jaugeages de crue basés sur des mesures de vitesse en surface (saumon de surface, vélocimètre radar, analyse de séquences d'images), l'incertitude accrue sur la mesure de vitesse et son intégration à l'aide d'un coefficient de vitesse rend encore plus réduit l'intérêt d'un grand nombre de verticales. La nouvelle méthode d'analyse des incertitudes peut ici aussi permettre de définir la meilleure stratégie à adopter.

\section{CONCLUSIONS}

Une nouvelle méthode pour calculer les incertitudes sur les débits jaugés par exploration du champ des vitesses a été développée à partir de la méthode de la norme ISO 748 
dont elle cherche à pallier les principaux défauts. Les cas variés de jaugeage sur lesquels elle a été testée ont confirmé qu'elle fournit des résultats plus cohérents pour les jaugeages moins «standard » (sections à faible rapport d'aspect) et que les incertitudes liées à l'échantillonnage spatial des vitesses et profondeurs, ainsi que celles liées à l'extrapolation des débits non mesurés, sont distribuées de façon réaliste. Les budgets d'incertitude obtenus sont utiles pour quantifier l'efficacité du processus de mesure face à chaque situation de jaugeage spécifique.

En perspective, la méthode pourra être améliorée en précisant les composantes d'incertitude liées à l'instrumentation et à l'environnement de mesure, notamment en croisant les résultats avec ceux des essais interlaboratoires. Pour sa validation et son amélioration, la méthode nécessite encore d'être confrontée au plus large jeu de jaugeages possible, surtout lorsque l'incertitude peut être estimée par une autre méthode. Il est prévu que le calcul soit implémenté dans le logiciel BAREME [Bechon et al. 2013] et dans le logiciel de dépouillement de la Société du Canal de Provence, ce qui facilitera les tests et le transfert opérationnel.

\section{REMERCIEMENTS}

Le développement de la méthode $\mathrm{Q}+$ et son intégration dans BAREME ont été soutenus par Irstea et le SCHAPI. La méthode a bénéficié des échanges avec les hydromètres qui ont fourni des jeux de données tests, en particulier José Ribot-Bruno (Irstea Montpellier), qui est à l'origine du questionnement sur les limites de la méthode ISO 748 pour les jaugeages atypiques.

\section{RÉFÉRENCES}

Bechon P.-M., Le Coz J., Leleu I., Renard B. (2013) - Des outils du réseau Etat en hydrométrie et leur ouverture aux notions d'incertitude. Actes du congrès SHF : «Hydrométrie 2013», Paris, 15-16 mai 2013

Boiten W. (2000) - Hydrometry. Francis and Taylor Publishers

CARTer R.W., ANDERson I.E. (1963) - Accuracy of current meter measurements. Journal of the Hydraulics Division Proceeding, ASCE. 89(HY4) : 105-115

Dramais G., Blanquart B., Le Coz J., Pierrefeu G., Hauet A., Atmane D., Pobanz K. (2014) — Les essais interlaboratoires en hydrométrie méthodologie et cas d'applications. La Houille blanche. 5

Forray N. ET Al. (1998) - Charte qualité de l'hydrométrie, Code de bonnes pratiques, Ministère de l'aménagement du territoire et de l'environnement. $55 \mathrm{p}$

Fulford J., SAuer V. (1986) - Comparison of velocity interpolation methods for computing open-channel discharge. US Geological Survey water-supply. 2290 : 139-144

Fulford J.M., Thibodeaux K.G., Kaehrle W.R. (1994) Comparison of current meters used for stream gaging. Fundamentals and Advancements in Hydraulic Measurements and Experimentation, August 1-5, 1994. New York, NY: American Society of Civil Engineers. 376-385
GuM. (2008) - Evaluation of measurement data - Guide to the expression of uncertainty in measurement, JCGM member organizations. BIPM, IEC, IFCC, ILAC, ISO, IUPAC, IUPAP and OIML (disponible en français et anglais sur le site internet du BIPM : http://www.bipm.org/fr/publications/guides/ gum.html)

Herschy R.W. (1999) - Hydrometry, Principles and Practices. 2nd ed. Wiley

HeRsCHY R.W. (2002) - The uncertainty in a current meter measurement. Flow Measurement and Instrumentation. 13 : 281-284

Iso/TR 9823. (1990) — Liquid flow measurement in open channels. Velocity-area method using a restricted number of verticals. Geneva (Switzerland): International Organization for Standardization

Iso/Ts 25377. (2007) - Hydrometric Uncertainty Guidance (HUG) Geneva (Switzerland): International Organization for Standardization

Iso 748. (2007) - Hydrometry-measurement of liquid flow in open channels using current-meters or floats. Geneva (Switzerland): International Organization for Standardization

JONES B.E. (1916) — A method of correcting river discharge for a changing stage. Water supply paper 375. US Geological Survey. 375 : p. 117-130

KIANG J.E., Cohn T.A., Mason R.R. (2009) — Quantifying uncertainty in discharge measurements: a new approach. World Environmental and Water Resources Congress 2009: Great Rivers. 342 : p. 5924-5931

Le Coz J., Camenen B., Dramais G., Ferry M., Rosique J.-L., Ribot-Bruno J. (2011) - Contrôle des débits réglementaires. Application de l'article L. 214-18 du Code de l'environnement, Guide pratique Onema/Cemagref. $128 \mathrm{p}$

Le Coz J., Camenen B., Peyrard X., Dramais G. (2012a) Uncertainty in open-channel discharges measured with the velocity-area method. Flow Measurement and Instrumentation. $26: 18-29$

Le Coz J., Camenen B., Peyrard X., Dramais G. (2014) Erratum to "Uncertainty in open-channel discharges measured with the velocity-area method [Flow Meas. Instrum. 26 (2012) 18-29]". - Flow Measurement and Instrumentation (published online DOI: 10.1016/j.flowmeasinst.2014.07.004)

Le Coz J., Pobanz K., Faure J.-B., Pierrefeu G., Choquette Y. (2012b) - Stage-discharge hysteresis evidenced by multi-ADCP measurements. Actes du congrès RiverFlow2012, 5-7 septembre 2012, San José, Costa Rica. p. 1277-1283

NF EN Iso 748 (2009) - Hydrométrie - Mesurage du débit des liquides dans les canaux découverts au moyen de moulinets ou de flotteurs, ISO. $50 \mathrm{p}$

Olivier A., Pierrefeu G., Scotti M., Blanquart B. (2009) - Incertitude des mesures de débit réalisées à l'ADCP. La Houille Blanche. 3 : 132-137

Pelletier P.M. (1988) - Uncertainties in the single determination of river discharge: a literature review

Canadian Journal of Civil Engineering. 15 : 834-850

RANTZ S.E. (1982) - Measurement and computation of streamflow. Vol. 1, Measurement of stage and discharge. Water-Supply. Washington: US Geological Survey. Paper 2175

SAUER V.B. (1992) - Determination of error in individual discharge measurements. USGS Open-File Report. 92-144 